\title{
On the quadratic Lagrange spectrum
}

\author{
Yann Bugeaud
}

\begin{abstract}
We study the quadratic Lagrange spectrum defined by Parkkonen and Paulin by considering the approximation by elements of the orbit of a given real quadratic irrational number for the action by homographies and anti-homographies of $P S L_{2}(\mathbf{Z})$ on $\mathbf{R} \cup\{\infty\}$. Our approach is based on the theory of continued fractions.
\end{abstract}

\section{Introduction}

We use throughout the superscript ${ }^{\sigma}$ to denote the Galois conjugate of a quadratic number. For every real quadratic irrational number $\alpha$, Parkkonen and Paulin $[9,10]$ introduced the quantity

$$
h(\alpha):=\frac{2}{\left|\alpha-\alpha^{\sigma}\right|},
$$

which may be viewed as a measure of the complexity of $\alpha$. As noted in [9], this quantity behaves in a very different way from the naïve height of $\alpha$ (the naïve height of an algebraic number is the maximum of the absolute values of the coefficients of its minimal defining polynomial over the rational integers), a notion which is commonly used in Diophantine approximation; see e.g. [5].

Let $\alpha_{0}$ be a fixed real quadratic irrational number and

$$
\mathcal{E}_{\alpha_{0}}=P S L_{2}(\mathbf{Z}) \cdot\left\{\alpha_{0}, \alpha_{0}^{\sigma}\right\}
$$

its orbit for the action by homographies and anti-homographies of $P S L_{2}(\mathbf{Z})$ on $\mathbf{R} \cup\{\infty\}$. In other words, $\mathcal{E}_{\alpha_{0}}$ is the set of quadratic numbers whose continued fraction expansion is ultimately periodic with the same period as $\alpha_{0}$ or as $\alpha_{0}^{\sigma}$.

For a real number $\xi$ not in $\mathbf{Q} \cup \mathcal{E}_{\alpha_{0}}$, Parkkonen and Paulin [9, 10] defined by

$$
c_{\alpha_{0}}(\xi):=\liminf _{\alpha \in \mathcal{E}_{\alpha_{0}}:\left|\alpha-\alpha^{\sigma}\right| \rightarrow 0} 2 \frac{|\xi-\alpha|}{\left|\alpha-\alpha^{\sigma}\right|}=\liminf _{\alpha \in \mathcal{E}_{\alpha_{0}}:\left|\alpha-\alpha^{\sigma}\right| \rightarrow 0}|\xi-\alpha| \cdot h(\alpha)
$$

2000 Mathematics Subject Classification : 11J06, 11J83. Keywords: Lagrange spectrum, continued fractions. 
the approximation constant $c_{\alpha_{0}}(\xi)$ of $\xi$ by elements of $\mathcal{E}_{\alpha_{0}}$ and they proved that $c_{\alpha_{0}}(\xi)$ is always finite. Observe that it follows immediately from (1.2) that

$$
c_{\alpha_{0}+k}\left(\xi+k^{\prime}\right)=c_{\alpha_{0}}(\xi)
$$

for every integers $k$ and $k^{\prime}$.

Furthermore, Parkkonen and Paulin [9, 10] defined the quadratic Lagrange spectrum of $\left(\alpha_{0}, P S L_{2}(\mathbf{Z})\right)$ by

$$
S p_{\alpha_{0}}:=\left\{c_{\alpha_{0}}(\xi): \xi \in \mathbf{R} \backslash\left(\mathbf{Q} \cup \mathcal{E}_{\alpha_{0}}\right)\right\} .
$$

Among other results, they showed that $S p_{\alpha_{0}}$ is a closed subset of $[0,(1+\sqrt{2}) \sqrt{3}]$. They also claimed that, in the special case when $\alpha_{0}$ is the Golden Ratio $\varphi:=(1+\sqrt{5}) / 2$, the maximum $K_{\alpha_{0}}$ of $S p_{\alpha_{0}}$ is equal to $1-1 / \sqrt{5}$. There is a slight overlook in the proof they proposed, but, fortunately, it can be fixed [11], and the exact value of $K_{(1+\sqrt{5}) / 2}$ is $-1+3 / \sqrt{5}=0.341 \ldots$ Apparently, their method does not give the exact value of $K_{\alpha_{0}}$ for a general real quadratic number $\alpha_{0}$.

The purpose of this note is to present a number theoretical interpretation of the approximation constant $c_{\alpha_{0}}(\xi)$ by means of the theory of continued fractions. We are then able, in principle, to compute $K_{\alpha_{0}}$ for every quadratic irrational number $\alpha_{0}$, although this computation is in general not an easy task. Our main new result states that the quadratic Lagrange spectrum is always contained in $[0,1 / 2]$.

Theorem 1.1. For any real quadratic irrational number $\alpha_{0}$, the maximum $K_{\alpha_{0}}$ of $S p_{\alpha_{0}}$ satisfies

$$
0<K_{\alpha_{0}} \leq \frac{1}{2}
$$

If the continued fraction expansion of $\alpha_{0}$ terminates in an infinite string of digits 1 , then

$$
K_{\alpha_{0}}=K_{(1+\sqrt{5}) / 2}=\frac{3}{\sqrt{5}}-1=0.341 \ldots
$$

Furthermore,

$$
\lim _{m \rightarrow+\infty} K_{\left(m+\sqrt{m^{2}+4}\right) / 2}=\frac{3}{\sqrt{5}}-1 .
$$

Like in $[9,11]$, our proof also gives that $K_{(1+\sqrt{5}) / 2}$ is not an isolated point in the spectrum $S p_{(1+\sqrt{5}) / 2}$.

We believe that $-1+3 / \sqrt{5}$ is a common upper bound for all the values $K_{\varphi_{0}}$. This seems to be, however, quite difficult to confirm.

The present note is organized as follows. Various results on continued fraction expansions are stated and proved in Section 2. They are applied to estimate the quantity $h(\alpha)$ defined in (1.1) and, in Section 3, to the proof of Theorem 1.1. We first explain how elementary arguments allow us to show that $K_{\alpha_{0}}$ is always at most equal to 4 (thus, slightly improving the upper bound $(1+\sqrt{2}) \sqrt{3}$ obtained in [9]). Then, we refine our analysis to get the upper bound $1 / 2$. Several remarks and additional results on the quantity $h(\alpha)$ are gathered in Section 4. 
Throughout, we use the notation

$$
\left[a_{0} ; a_{1}, a_{2} \ldots, a_{r}, \overline{a_{r+1}, \ldots, a_{r+s}}\right]:=a_{0}+\frac{1}{a_{1}+\frac{1}{a_{2}+\frac{1}{\ldots}}}
$$

to indicate that the block of partial quotients $a_{r+1}, \ldots, a_{r+s}$ is repeated infinitely many times.

We recall that an irrational real number $\alpha$ is quadratic if, and only if, its continued fraction expansion is ultimately periodic, that is, is of the form

$$
\alpha=\left[a_{0} ; a_{1}, \ldots, a_{r}, \overline{b_{1}, \ldots, b_{s}}\right] .
$$

When we express $\alpha$ as in (1.4) we tacitly assume that $s$ is minimal and that $a_{r} \neq b_{s}$. We call $b_{1}, \ldots, b_{s}$ the shortest periodic part in the continued fraction expansion of $\alpha$.

\section{Auxiliary lemmas on continued fraction expansions}

We assume that the reader is familiar with the theory of continued fractions. Good references include $[12,8]$ and the first chapters of $[14]$ and $[5]$.

Let us simply recall that two irrational real numbers $\alpha, \beta$ are called equivalent if there exist rational integers $a, b, c, d$ with $a d-b c= \pm 1$ such that

$$
\alpha=\frac{a \beta+b}{c \beta+d}
$$

It is easily shown (see [12], page 65) that $\alpha$ and $\beta$ are equivalent if, and only if, the tails of their continued fraction expansions coincide.

Our first lemma establishes a link between the quantity $h(\alpha)$ defined in (1.1) and the preperiod of the continued fraction expansion of the real quadratic number $\alpha$.

Lemma 2.1. Let $\alpha$ be a quadratic real number with ultimately periodic continued fraction expansion

$$
\alpha=\left[a_{0} ; a_{1}, \ldots, a_{r}, \overline{b_{1}, \ldots, b_{s}}\right],
$$

with $r \geq 3$ and $s \geq 1$, and denote by $\alpha^{\sigma}$ its Galois conjugate. Let $\left(p_{\ell} / q_{\ell}\right)_{\ell \geq 1}$ denote the sequence of convergents to $\alpha$. Set $B:=\max \left\{b_{1}, \ldots, b_{s}, 2\right\}$. If $a_{r} \neq b_{s}$, then we have

$$
\frac{1}{2 q_{r}^{2}} \leq\left|\alpha-\alpha^{\sigma}\right| \leq \frac{8 B^{3}}{q_{r}^{2}},
$$

thus,

$$
q_{r}^{2} /\left(4 B^{3}\right) \leq h(\alpha) \leq 4 q_{r}^{2} .
$$

This is essentially Lemma 6.1 from [6]. For the sake of completeness, we reproduce its proof. 
Proof. A theorem of Galois (see [12], page 83) states that the Galois conjugate of

$$
\tau:=\left[b_{1} ; \overline{b_{2}, \ldots, b_{s}, b_{1}}\right]
$$

is the quadratic number

$$
\tau^{\sigma}=-\left[0 ; \overline{b_{s}, \ldots, b_{2}, b_{1}}\right] .
$$

Since, by Theorem 1.7 of [5], we have

$$
\alpha=\frac{p_{r} \tau+p_{r-1}}{q_{r} \tau+q_{r-1}} \quad \text { and } \quad \alpha^{\sigma}=\frac{p_{r} \tau^{\sigma}+p_{r-1}}{q_{r} \tau^{\sigma}+q_{r-1}}
$$

we get

$$
\begin{aligned}
\left|\alpha-\alpha^{\sigma}\right| & =\frac{\tau-\tau^{\sigma}}{\left(q_{r} \tau+q_{r-1}\right) \cdot\left|q_{r} \tau^{\sigma}+q_{r-1}\right|} \\
& \leq \frac{\tau+1}{\tau q_{r} \cdot\left|q_{r} \tau^{\sigma}+q_{r-1}\right|} \leq \frac{2}{q_{r} \cdot\left|q_{r} \tau^{\sigma}+q_{r-1}\right|}
\end{aligned}
$$

Likewise, using $\left|q_{r} \tau^{\sigma}+q_{r-1}\right| \leq q_{r}$, we obtain

$$
\left|\alpha-\alpha^{\sigma}\right| \geq \frac{\tau-\tau^{\sigma}}{(\tau+1) q_{r}^{2}} \geq \frac{1}{2 q_{r}^{2}} .
$$

Since $a_{r} \neq b_{s}$ the mirror formula (see Lemma $3 \mathrm{~F}$ of [14])

$$
q_{r-1} / q_{r}=\left[0 ; a_{r}, a_{r-1}, \ldots, a_{1}\right]
$$

implies that

$$
\left|q_{r} \tau^{\sigma}+q_{r-1}\right|=\left|\left[0 ; a_{r}, a_{r-1}, \ldots, a_{1}\right]-\left[0 ; \overline{b_{s}, \ldots, b_{2}, b_{1}}\right]\right| \cdot q_{r}
$$

If $a_{r} \geq 2 B$, then one gets

$$
\left|q_{r} \tau^{\sigma}+q_{r-1}\right| \geq\left(\frac{1}{B+1}-\frac{1}{2 B}\right) q_{r} \geq \frac{q_{r}}{6 B} .
$$

Otherwise, if $a_{r}<b_{s}$, then $a_{r} \leq B-1$ and an easy computation shows that

$$
\begin{aligned}
\left|q_{r} \tau^{\sigma}+q_{r-1}\right| & \geq\left(\frac{1}{a_{r}+1}-\frac{1}{b_{s}+1 /\left(b_{s-1}+1\right)}\right) \cdot q_{r} \\
& \geq \frac{q_{r}}{\left(a_{r}+1\right)\left(b_{s}\left(b_{s-1}+1\right)+1\right)} \geq \frac{q_{r}}{3 B^{3}},
\end{aligned}
$$

while, if $b_{s}<a_{r} \leq 2 B-1$, then the similar estimate

$$
\left|q_{r} \tau^{\sigma}+q_{r-1}\right| \geq \frac{q_{r}}{a_{r} b_{s}(2 B)} \geq \frac{q_{r}}{4 B^{3}}
$$

holds. By (2.2) and (2.3), this completes the proof of the lemma.

Our second auxiliary lemma is very close to Lemma 5 from [1] (see also Lemma 5.5 from [3]). 
Lemma 2.2. Let $B$ be a positive integer. Let $\alpha=\left[0 ; a_{1}, a_{2}, \ldots\right]$ and $\beta=\left[0 ; b_{1}, b_{2}, \ldots\right]$ be real numbers. Assume that there exists a positive integer $n$ such that $a_{i}=b_{i}$ for any $i=1, \ldots, n$ and $a_{n+1} \neq b_{n+1}$. Then, we have

$$
|\alpha-\beta| \geq \frac{1}{12 q_{n+1}^{2} \max \left\{b_{n+2}+1, b_{n+3}+2\right\}},
$$

where $\left(p_{\ell} / q_{\ell}\right)_{\ell \geq 1}$ is the sequence of convergents to $\beta$.

Proof. Set $\alpha^{\prime}=\left[a_{n+1} ; a_{n+2}, \ldots\right]$ and $\beta^{\prime}=\left[b_{n+1} ; b_{n+2}, \ldots\right]$. Since $a_{n+1} \neq b_{n+1}$, a rapid calculation shows that

$$
\left|\alpha^{\prime}-\beta^{\prime}\right| \geq \min \left\{\frac{1}{b_{n+2}+1}, \frac{1}{b_{n+3}+2}\right\} .
$$

Using that the first $n$ partial quotients of $\alpha$ and $\beta$ are assumed to be the same, we get

$$
\alpha=\frac{p_{n} \alpha^{\prime}+p_{n-1}}{q_{n} \alpha^{\prime}+q_{n-1}} \quad \text { and } \quad \beta=\frac{p_{n} \beta^{\prime}+p_{n-1}}{q_{n} \beta^{\prime}+q_{n-1}},
$$

thus,

$$
|\alpha-\beta|=\left|\frac{p_{n} \alpha^{\prime}+p_{n-1}}{q_{n} \alpha^{\prime}+q_{n-1}}-\frac{p_{n} \beta^{\prime}+p_{n-1}}{q_{n} \beta^{\prime}+q_{n-1}}\right|=\left|\frac{\alpha^{\prime}-\beta^{\prime}}{\left(q_{n} \alpha^{\prime}+q_{n-1}\right)\left(q_{n} \beta^{\prime}+q_{n-1}\right)}\right| .
$$

Note that $q_{n} \beta^{\prime}+q_{n-1} \leq 2 q_{n+1}$.

If $\alpha^{\prime} \leq 2 \beta^{\prime}+1 \leq 2 b_{n+1}+3$, then $q_{n} \alpha^{\prime}+q_{n-1} \leq 2\left(b_{n+1}+2\right) q_{n}$ and, by $(2.5)$ and (2.6), we get

$$
|\alpha-\beta| \geq \frac{1}{4\left(b_{n+1}+2\right) q_{n} q_{n+1} \max \left\{b_{n+2}+1, b_{n+3}+2\right\}} .
$$

If $\alpha^{\prime} \geq 2 \beta^{\prime}+1$, then $\left|\alpha^{\prime}-\beta^{\prime}\right| \geq\left(\alpha^{\prime}+1\right) / 2$ and

$$
|\alpha-\beta| \geq \frac{1}{4 q_{n} q_{n+1}} .
$$

Since $\left(b_{n+1}+2\right) q_{n} \leq 3 q_{n+1}$, the estimate (2.4) follows from (2.7) and (2.8).

We display an easy consequence of Lemma 2.2 .

Corollary 2.3. Let

$$
\tau=\left[0 ; \overline{b_{1}, b_{2} \ldots, b_{s}}\right]
$$

be a quadratic number whose shortest periodic part is $b_{1}, \ldots, b_{s}$. Let

$$
\xi=\left[a_{0} ; a_{1}, a_{2}, \ldots\right]
$$

be an irrational real number not in $\mathcal{E}_{\tau}$. For positive integers $r$ and $t$, let $a_{1}^{\prime}, \ldots, a_{t}^{\prime}$ be positive integers with $a_{1}^{\prime} \neq a_{r+1}$ and $a_{t}^{\prime} \neq b_{s}$, and set

$$
\alpha:=\left[a_{0} ; a_{1}, a_{2}, \ldots, a_{r}, a_{1}^{\prime}, \ldots, a_{t}^{\prime}, \overline{b_{1}, \ldots, b_{s-1}, b_{s}}\right] .
$$


Putting $B:=\max \left\{b_{1}, \ldots, b_{s}, 2\right\}$, we have

$$
|\xi-\alpha| \cdot h(\alpha) \geq \frac{1}{96 B^{3}(B+1)^{2}} .
$$

Furthermore, $|\xi-\alpha| \cdot h(\alpha) \geq 1$ if $t \geq 9.6+4.4 \log B$.

Proof. Let $\left(p_{\ell} / q_{\ell}\right)_{\ell \geq 1}$ denote the sequence of convergents to $\alpha$. We deduce from Lemma 2.2 that

$$
|\xi-\alpha| \geq \frac{1}{24 q_{r+1} q_{r+3}}
$$

and from Lemma 2.1 that

$$
h(\alpha) \geq q_{r+t}^{2} /\left(4 B^{3}\right) .
$$

This gives

$$
|\xi-\alpha| \cdot h(\alpha) \geq 1 /\left(96 B^{3}\right)
$$

if $t \geq 3$. If $t=1$, then $q_{r+3} \leq(B+1)^{2} q_{r+1}$ and we obtain the upper bound

$$
|\xi-\alpha| \cdot h(\alpha) \geq \frac{1}{96 B^{3}(B+1)^{2}}
$$

If $t=2$, then, using $q_{r+3} \leq(B+1) q_{r+2}$, we see that (2.12) holds. This proves $(2.9)$.

An easy induction shows that $q_{\ell} \geq \sqrt{2}^{\ell-h-1} q_{h}$ for $\ell>h \geq 1$. Consequently, for $t \geq 4$, we deduce from (2.10) and (2.11) that

$$
|\xi-\alpha| \cdot h(\alpha) \geq \frac{2^{t}}{768 B^{3}}
$$

This implies that $|\xi-\alpha| \cdot h(\alpha) \geq 1$ if $t \geq 9.6+4.4 \log B$.

For positive integers $a_{1}, \ldots, a_{\ell}$, denote by $K_{\ell}\left(a_{1}, \ldots, a_{\ell}\right)$ the denominator of the rational number $\left[0, a_{1}, \ldots, a_{\ell}\right]$. It is commonly called the continuant of $a_{1}, \ldots, a_{\ell}$. A proof of the next lemma can be found on page 15 of [12].

Lemma 2.4. For any positive integers $a_{1}, \ldots, a_{\ell}$ and any integer $k$ with $1 \leq k \leq \ell-1$, we have

$$
K_{\ell}\left(a_{1}, \ldots, a_{\ell}\right)=K_{\ell}\left(a_{\ell}, \ldots, a_{1}\right)
$$

and

$$
\begin{aligned}
K_{k}\left(a_{1}, \ldots, a_{k}\right) \cdot K_{\ell-k}\left(a_{k+1}, \ldots, a_{\ell}\right) & \leq K_{\ell}\left(a_{1}, \ldots, a_{\ell}\right) \\
& \leq 2 K_{k}\left(a_{1}, \ldots, a_{k}\right) \cdot K_{\ell-k}\left(a_{k+1}, \ldots, a_{\ell}\right) .
\end{aligned}
$$




\section{Proof of Theorem 1.1}

\subsection{Preliminaries.}

Let $b_{1}, \ldots, b_{s}$ be the (shortest) periodic part in the continued fraction expansion of $\alpha_{0}$ and set

$$
\tau=\left[b_{1} ; \overline{b_{2}, \ldots, b_{s}, b_{1}}\right] .
$$

For $j=1, \ldots, s$, set

$$
\tau_{j}:=\left[b_{j} ; \overline{b_{j+1}, \ldots, b_{j-1}, b_{j}}\right]
$$

and

$$
\tau_{j}^{\prime}=\left[b_{j-1} ; \overline{b_{j-2}, b_{j-3}, \ldots, b_{j}, b_{j-1}}\right],
$$

where the indices are understood modulo $s$. Observe that $\tau=\tau_{1}$ and $\mathcal{E}_{\tau}=\mathcal{E}_{\tau_{1}}=\ldots=$ $\mathcal{E}_{\tau_{s}}=\mathcal{E}_{\tau_{1}^{\prime}}=\ldots=\mathcal{E}_{\tau_{s}^{\prime}}=\mathcal{E}_{\alpha_{0}}$. Furthermore, by the theorem of Galois already mentioned at the beginning of the proof of Lemma 2.1, we have

$$
\tau_{j}^{\sigma}=-\left[0 ; \overline{b_{j-1}, b_{j-2}, \ldots, b_{j+1}, b_{j}}\right]=-1 / \tau_{j}^{\prime},
$$

for $j=1, \ldots, s$.

Let

$$
\xi=\left[0 ; a_{1}, a_{2}, \ldots\right]
$$

be an irrational real number not in $\mathcal{E}_{\tau}$, which we wish to approximate by numbers from $\mathcal{E}_{\tau}$. Let $\left(p_{\ell} / q_{\ell}\right)_{\ell \geq 1}$ denote the sequence of convergents to $\xi$. By (1.3), our assumption that $\xi$ is in $(0,1)$ is not restrictive.

3.2. An observation.

For $r \geq 1$, the quadratic number

$$
\alpha_{r}:=\left[0 ; a_{1}, a_{2}, \ldots, a_{r}, \overline{b_{1}, \ldots, b_{s-1}, b_{s}}\right]=\left[0 ; a_{1}, a_{2}, \ldots, a_{r}, \tau\right]
$$

is a quite good approximation to $\xi$ in $\mathcal{E}_{\tau}$ and

$$
\ell_{\tau}(\xi):=\liminf _{r \rightarrow+\infty}\left|\xi-\alpha_{r}\right| \cdot h\left(\alpha_{r}\right)
$$

is greater than or equal to $c_{\tau}(\xi)$.

By taking also into account the circular permutations of the periodic part of the continued fraction expansion of $\tau$ and of that of $\tau^{\sigma}$, we see that

$$
c_{\tau}(\xi) \leq \min _{1 \leq j \leq s} \min \left\{\ell_{\tau_{j}}(\xi), \ell_{\tau_{j}^{\prime}}(\xi)\right\}
$$

At first sight, we may expect that equality holds in (3.1). In order to confirm this, we would need to estimate the quantities $|\xi-\alpha| \cdot h(\alpha)$ for quadratic numbers $\alpha$ of the form

$$
\left[0 ; a_{1}, a_{2}, \ldots, a_{r}, a_{1}^{\prime}, \ldots, a_{t}^{\prime}, \tau_{j}\right] \quad \text { and } \quad\left[0 ; a_{1}, a_{2}, \ldots, a_{r}, a_{1}^{\prime}, \ldots, a_{t}^{\prime}, \tau_{j}^{\prime}\right]
$$


where $1 \leq j \leq s, t \geq 1$ and $a_{1}^{\prime}, \ldots, a_{t}^{\prime}$ are positive integers. By Corollary 2.3, we know already that $|\xi-\alpha| \cdot h(\alpha) \geq 1$ if $t$ is sufficiently large in terms of $b_{1}, \ldots, b_{s}$. However, even for small values of $t$, it is difficult to estimate precisely $|\xi-\alpha| \cdot h(\alpha)$ for a general quadratic number $\tau$.

Actually, we will see below that, unlike what would be expected, there exist quadratic numbers $\tau$ for which we do not have equality in (3.1).

3.3. A first upper bound.

Since $\xi$ and $\alpha_{r}$ have the same first $r$ partial quotients, we deduce from Theorem 1.7 of $[5]$ that

$$
\left|\xi-\alpha_{r}\right| \leq \frac{1}{q_{r}^{2}}
$$

It then follows from (2.1) that

$$
\left|\xi-\alpha_{r}\right| \cdot h\left(\alpha_{r}\right) \leq 4
$$

which gives that

$$
c_{\alpha_{0}}(\xi) \leq 4
$$

We recover, with a fairly simple proof, that the quantities $K_{\alpha_{0}}$ are always finite and, even, absolutely bounded. The upper bound 4 is slightly smaller than the value $(1+\sqrt{2}) \sqrt{3}=$ $4.18 \ldots$ obtained in [9]. However, our method using continued fraction expansions allows us to improve further this upper bound.

\subsection{Preliminary calculation.}

Since, by Theorem 1.7 of [5], we have

$$
\alpha_{r}=\frac{p_{r} \tau+p_{r-1}}{q_{r} \tau+q_{r-1}} \quad \text { and } \quad \alpha_{r}^{\sigma}=\frac{p_{r} \tau^{\sigma}+p_{r-1}}{q_{r} \tau^{\sigma}+q_{r-1}}
$$

we deduce that

$$
\left|\alpha_{r}-\alpha_{r}^{\sigma}\right|=\frac{\tau-\tau^{\sigma}}{\left(q_{r} \tau+q_{r-1}\right) \cdot\left|q_{r} \tau^{\sigma}+q_{r-1}\right|} .
$$

Observe now that

$$
\begin{aligned}
\left|\xi-\alpha_{r}\right| & =\left|\frac{p_{r}\left[a_{r+1} ; a_{r+2}, \ldots\right]+p_{r-1}}{q_{r}\left[a_{r+1} ; a_{r+2}, \ldots\right]+q_{r-1}}-\frac{p_{r} \tau+p_{r-1}}{q_{r} \tau+q_{r-1}}\right| \\
& =\frac{\left|\tau-\left[a_{r+1} ; a_{r+2}, \ldots\right]\right|}{\left(q_{r}\left[a_{r+1} ; a_{r+2}, \ldots\right]+q_{r-1}\right) \cdot\left(q_{r} \tau+q_{r-1}\right)}
\end{aligned}
$$

and, using (3.2),

$$
2 \frac{\left|\xi-\alpha_{r}\right|}{\left|\alpha_{r}-\alpha_{r}^{\sigma}\right|}=\frac{2\left|\tau-\left[a_{r+1} ; a_{r+2}, \ldots\right]\right| \cdot\left|q_{r} \tau^{\sigma}+q_{r-1}\right|}{\left(\tau-\tau^{\sigma}\right) \cdot\left(q_{r}\left[a_{r+1} ; a_{r+2}, \ldots\right]+q_{r-1}\right)} .
$$


Set

$$
d_{r}:=\frac{q_{r}}{q_{r-1}}=\left[a_{r} ; a_{r-1}, \ldots, a_{1}\right]
$$

and

$$
D_{r}:=\left[a_{r+1} ; a_{r+2}, \ldots\right] .
$$

The right hand side of (3.3) then becomes

$$
\frac{2}{\tau-\tau^{\sigma}} \cdot \frac{\left|\tau-D_{r}\right| \cdot\left|\tau^{\sigma} d_{r}+1\right|}{1+d_{r} D_{r}}
$$

We stress that, to establish (3.4) we do not have used that the continued fraction expansion of $\tau$ is purely periodic.

Define

$$
\tau^{\prime}:=-1 / \tau^{\sigma}=1 /\left|\tau^{\sigma}\right|=\left[b_{s} ; \overline{b_{s-1}, \ldots, b_{1}, b_{s}}\right] .
$$

Then, (3.4) becomes

$$
\frac{2}{\tau \tau^{\prime}+1} \cdot \frac{\left|\tau-D_{r}\right| \cdot\left|\tau^{\prime}-d_{r}\right|}{1+d_{r} D_{r}}
$$

Keeping the notation of Subsection 3.1, this proves that

$$
\ell_{\tau_{j}}(\xi)=\liminf _{r \rightarrow+\infty} \frac{2}{\tau_{j} \tau_{j}^{\prime}+1} \cdot \frac{\left|\tau_{j}-D_{r}\right| \cdot\left|\tau_{j}^{\prime}-d_{r}\right|}{1+d_{r} D_{r}}
$$

for $j=1, \ldots, s$. Similarly, we obtain

$$
\ell_{\tau_{j}^{\prime}}(\xi)=\liminf _{r \rightarrow+\infty} \frac{2}{\tau_{j} \tau_{j}^{\prime}+1} \cdot \frac{\left|\tau_{j}^{\prime}-D_{r}\right| \cdot\left|\tau_{j}-d_{r}\right|}{1+d_{r} D_{r}} .
$$

Consequently, the quantity

$$
c_{\tau}^{\prime}(\xi):=\min _{1 \leq j \leq s} \frac{2}{\tau_{j} \tau_{j}^{\prime}+1} \cdot \liminf _{r \rightarrow+\infty} \frac{\min \left\{\left|\tau_{j}-D_{r}\right| \cdot\left|\tau_{j}^{\prime}-d_{r}\right|,\left|\tau_{j}^{\prime}-D_{r}\right| \cdot\left|\tau_{j}-d_{r}\right|\right\}}{1+d_{r} D_{r}}
$$

is greater than or equal to $c_{\tau}(\xi)$. As explained below (3.1), it could be strictly greater than $c_{\tau}(\xi)$, since the quadratic numbers we have considered are special elements of the orbit of $\alpha_{0}$ and of that of $\alpha_{0}^{\sigma}$.

3.5. A refined upper bound.

Our goal is to establish that the quantity $c_{\tau}^{\prime}(\xi)$ defined in (3.5) is always at most equal to $1 / 2$.

First, we assume that neither $\tau$, nor $\xi$ is equivalent to the Golden Ratio $\varphi$. This means that both have infinitely many partial quotients at least equal to 2 . 
In particular, either infinitely many partial quotients of $\xi$ are at least equal to 3 , in which case $d_{r} D_{r} \geq 3$ for infinitely many $r$, or

$$
d_{r} D_{r} \geq\left(1+\frac{1}{3}\right) \cdot\left(2+\frac{1}{3}\right)=\frac{28}{9},
$$

for infinitely many $r$.

Since $\tau$ and $\xi$ are not equivalent to $\varphi$, we can suppose that $\tau^{\prime}>2$ and $D_{r}>2$. Furthermore, arguing as for (3.6), we can suppose that

$$
\tau \tau^{\prime} \geq 3
$$

If $\tau, \tau^{\prime}, d_{r}, D_{r}$ are all greater than 2 , then it follows from (3.5) that

$$
c_{\tau}^{\prime}(\xi) \leq \frac{2 \max \left\{\tau, D_{r}\right\} \cdot \max \left\{\tau^{\prime}, d_{r}\right\}}{\tau \tau^{\prime} d_{r} D_{r}} \leq \frac{1}{2}
$$

Assume that $\tau>2$ and $1<d_{r}<2$.

If $\tau \geq D_{r} / 2$, then $\left|\tau-D_{r}\right| \leq \tau$ and, since $\left|\tau^{\prime}-d_{r}\right| \leq \tau^{\prime}$, we get from (3.6) that

$$
c_{\tau}^{\prime}(\xi) \leq \frac{2}{1+d_{r} D_{r}} \leq \frac{1}{2}
$$

The same upper bound holds if $\tau^{\prime} \geq D_{r} / 2$, since then we have $\left|\tau-d_{r}\right| \leq \tau$. Consequently, we can assume that $D_{r} \geq 2 \tau$ and $D_{r} \geq 2 \tau^{\prime}$, thus,

$$
c_{\tau}^{\prime}(\xi) \leq \min \left\{\frac{2\left|\tau^{\prime}-d_{r}\right|}{d_{r}\left(1+\tau \tau^{\prime}\right)}, \frac{2\left|\tau-d_{r}\right|}{d_{r}\left(1+\tau \tau^{\prime}\right)}\right\}
$$

We get $c_{\tau}^{\prime}(\xi) \leq 1 / 2$ if $\tau \geq 4$ or $\tau^{\prime} \geq 4$. So, we can assume that $2<\tau, \tau^{\prime}<4$. If $\tau \leq \tau^{\prime}$, then

$$
c_{\tau}^{\prime}(\xi) \leq \frac{2(\tau-1)}{1+\tau \tau^{\prime}} \leq \frac{2(\tau-1)}{1+\tau \tau^{2}} \leq \frac{1}{2},
$$

and, by symmetry, the estimate $c_{\tau}^{\prime}(\xi) \leq 1 / 2$ also holds if $\tau^{\prime} \leq \tau$.

Assume that $1<\tau \leq 2$.

We get $\left|\tau-D_{r}\right| \leq D_{r}$. If $d_{r} \geq \tau^{\prime} / 2$, it then follows from (3.7) that

$$
c_{\tau}^{\prime}(\xi) \leq \frac{2}{\tau \tau^{\prime}+1} \leq \frac{1}{2}
$$

Using the symmetry, the same upper bound holds when $D_{r} \geq \tau^{\prime} / 2$. Consequently, we can assume that $\tau^{\prime} \geq 2 D_{r}$ and $\tau^{\prime} \geq 2 d_{r}$.

Arguing as above, we see that one can assume that $D_{r}$ and $d_{r}$ are both $<4$ and, moreover, that $1<d_{r}<2$. In particular, the partial quotients of $\xi$ belong to $\{1,2,3\}$. Arguing as in (3.6), this implies that $d_{r} \geq 5 / 4$ and $D_{r} \geq 9 / 4$. Furthermore, since $5 / 4 \leq$ $d_{r}<2$ and $1<\tau<2$, we get that $\left|\tau-d_{r}\right| \leq 3 \tau / 4$ and, consequently,

$$
c_{\tau}^{\prime}(\xi) \leq \min \left\{\frac{2\left|\tau-d_{r}\right|, 2\left|\tau-D_{r}\right|}{\tau\left(1+d_{r} D_{r}\right)}\right\} \leq \frac{2}{5}
$$


To summarize, we have established that, unless $\tau$ or $\xi$ is equivalent to the Golden Ratio, we always have

$$
c_{\tau}(\xi) \leq c_{\tau}^{\prime}(\xi) \leq 1 / 2
$$

3.6. When $\tau$ is the Golden Ratio.

Assume that $\tau=\varphi=(1+\sqrt{5}) / 2$. To determine $K_{\varphi}$, we need to find $\xi=\left[0 ; a_{1}, a_{2}, \ldots\right]$ for which $c_{\tau}(\xi)$ is as large as possible. Clearly, all the $a_{j}$ have to be taken very large and we then derive from (3.5) that

$$
c_{\varphi}^{\prime}(\xi) \leq \frac{2}{1+\varphi^{2}} \cdot \frac{d_{r} D_{r}}{1+d_{r} D_{r}} \leq 1-\frac{1}{\sqrt{5}}
$$

and $c_{\varphi}^{\prime}(\xi)$ can be arbitrarily close to $1-1 / \sqrt{5}$. Actually, it turns out that the approximations $\left[0 ; a_{1}, a_{2}, \ldots, a_{r}, \overline{1}\right]=\left[0 ; a_{1}, a_{2}, \ldots, a_{r}, \varphi\right]$ are not the closest ones to $\xi$ and that we gain by considering the approximations

$$
\left[0 ; a_{1}, a_{2}, \ldots, a_{r}, 2, \overline{1}\right]=\left[0 ; a_{1}, a_{2}, \ldots, a_{r}, 1+\varphi\right] .
$$

Indeed, (3.4) then becomes

$$
\frac{2}{\varphi-\varphi^{\sigma}} \cdot \frac{\left|1+\varphi-D_{r}\right| \cdot\left|\left(1+\varphi^{\sigma}\right) d_{r}+1\right|}{1+d_{r} D_{r}}
$$

and, since $d_{r}$ and $D_{r}$ are very large, we obtain the limiting value

$$
\frac{2\left(1+\varphi^{\sigma}\right)}{\varphi-\varphi^{\sigma}}=\frac{3}{\sqrt{5}}-1
$$

This shows that $c_{\varphi}(\xi)<-1+3 / \sqrt{5}$ for every irrational number $\xi$ not in $\mathcal{E}_{\varphi}$. Furthermore, we check that, for $\alpha=\left[0 ; a_{1}, a_{2}, \ldots, a_{r}, a_{1}^{\prime}, \ldots, a_{t}^{\prime}, \overline{1}\right]$, with $t \geq 1, a_{t}^{\prime} \neq 1, a_{1}^{\prime} \neq a_{r+1}$, and $a_{t}^{\prime} \neq 2$ if $t=1$, the quantity $|\xi-\alpha| \cdot h(\alpha)$ exceeds $-1+3 / \sqrt{5}$. To see this, setting

$$
\frac{p_{t}^{\prime}}{q_{t}^{\prime}}:=\left[a_{1}^{\prime} ; a_{2}^{\prime}, \ldots, a_{t}^{\prime}\right], \quad \frac{p_{t-1}^{\prime}}{q_{t-1}^{\prime}}:=\left[a_{1}^{\prime} ; a_{2}^{\prime}, \ldots, a_{t-1}^{\prime}\right] \quad \text { and } \quad \varphi_{t}:=\frac{p_{t}^{\prime} \varphi+p_{t-1}^{\prime}}{q_{t}^{\prime} \varphi+q_{t-1}^{\prime}}
$$

the formula (3.4) becomes

$$
\frac{2}{\varphi_{t}-\varphi_{t}^{\sigma}} \cdot \frac{\left|\varphi_{t}-D_{r}\right| \cdot\left|\varphi_{t}^{\sigma} d_{r}+1\right|}{1+d_{r} D_{r}}
$$

Again, since $d_{r}$ and $D_{r}$ are very large, we obtain the limiting value

$$
\frac{2\left|\varphi_{t}^{\sigma}\right|}{\varphi_{t}-\varphi_{t}^{\sigma}}
$$


Noticing that $\varphi_{t}-\varphi_{t}^{\sigma} \leq \varphi-\varphi^{\sigma}$ and $\left|\varphi_{t}^{\sigma}\right| \geq 1+\varphi^{\sigma}$, we see that the minimum of this quantity is attained for $\varphi_{t}=1+\varphi$.

Consequently, we have established that $K_{\varphi}=-1+3 / \sqrt{5}$. Moreover, setting

$$
\varphi_{m}:=[m ; \bar{m}]=\left(m+\sqrt{m^{2}+4}\right) / 2
$$

for $m \geq 1$, we see that $\varphi=\varphi_{1}$,

$$
c_{\varphi}\left(\varphi_{m}\right)<3 / \sqrt{5}-1 \text { and } \lim _{m \rightarrow+\infty} c_{\varphi}\left(\varphi_{m}\right)=3 / \sqrt{5}-1
$$

\subsection{When $\xi$ is the Golden Ratio.}

For $\xi=\varphi=(1+\sqrt{5}) / 2$, we have $D_{r}=\varphi$ and $d_{r}$ approaches $\varphi$ as $r$ tends to infinity. Since we are looking for large values of $c_{\tau}(\varphi)$ we can assume that $b_{1}, \ldots, b_{s}$ are all at least equal to 2 . It then follows from (3.5) that

$$
c_{\tau}^{\prime}(\varphi) \leq \frac{2}{1+\varphi^{2}} \cdot \frac{\tau \tau^{\prime}}{1+\tau \tau^{\prime}}<1-\frac{1}{\sqrt{5}}
$$

This establishes that $c_{\tau}(\varphi)$ is always smaller than $1-1 / \sqrt{5}$. We have considered the approximants $[1 ; 1, \ldots, 1, \tau]$, but, quite surprisingly, the approximants

$$
[1 ; 1, \ldots, 1, \tau-1]=\left[1 ; 1, \ldots, 1, b_{1}-1, \overline{b_{2}, \ldots, b_{s}, b_{1}}\right]
$$

give a better estimate. Indeed, by (3.4), we then have to consider

$$
\frac{2}{\tau-\tau^{\sigma}} \cdot \frac{|(\tau-1)-\varphi| \cdot\left|\left(\tau^{\sigma}-1\right) \varphi+1\right|}{1+\varphi^{2}},
$$

and, when $\tau$ and $1 /\left|\tau^{\sigma}\right|$ tend to infinity, the above quantity tends to

$$
\frac{2 \cdot|1-\varphi|}{1+\varphi^{2}}=\frac{3}{\sqrt{5}}-1
$$

Furthermore, since $|n \varphi+1| \geq|1-\varphi|$ for every integer $n$, the quantity $|\varphi-\alpha| \cdot h(\alpha)$ always exceeds $-1+3 / \sqrt{5}$.

Consequently, we have established that $c_{\tau}(\varphi)<-1+3 / \sqrt{5}$ and, moreover,

$$
\lim _{m \rightarrow+\infty} c_{\varphi_{m}}(\varphi)=3 / \sqrt{5}-1
$$

This shows that

$$
\lim _{m \rightarrow+\infty} K_{\varphi_{m}}=K_{\varphi}
$$

thus completing the proof of Theorem 1.1. 


\subsection{Some speculation.}

It seems that there are two extremal cases to determine the value of $K_{\tau}$ for a given real quadratic number $\tau$. A first one is when all the $a_{j}$ are large, and a second one when all the $a_{j}$ are equal to 1 , that is, when $\xi=\varphi$. This observation suggests that

$$
K_{\tau}:=\max \left\{c_{\tau}(\varphi), \lim _{m \rightarrow+\infty} c_{\tau}\left(\varphi_{m}\right)\right\}
$$

Using (3.4) and arguing as in Subsection 3.6, we also believe that

$$
\lim _{m \rightarrow+\infty} c_{\tau}\left(\varphi_{m}\right)=\min _{1 \leq j \leq s} \min \left\{\frac{2 \min \left\{\left|\tau_{j}^{\sigma}\right|, 1+\tau_{j}^{\sigma}\right\}}{\tau_{j}-\tau_{j}^{\sigma}}, \frac{2 \min \left\{\left|\tau_{j}^{\prime \sigma}\right|, 1+\tau_{j}^{\prime \sigma}\right\}}{\tau_{j}^{\prime}-\tau_{j}^{\prime \sigma}}\right\} .
$$

Since $\tau_{j}^{\prime}=-1 / \tau_{j}^{\sigma}$ for $j=1, \ldots, s$, the above formula can be rewritten as

$$
\lim _{m \rightarrow+\infty} c_{\tau}\left(\varphi_{m}\right)=\min _{1 \leq j \leq s} \frac{2 \min \left\{\left|\tau_{j}^{\sigma}\right|, 1+\tau_{j}^{\sigma},\left|\tau_{j}^{\sigma}\right|\left(\tau_{j}-1\right)\right\}}{\tau_{j}-\tau_{j}^{\sigma}} .
$$

Likewise, using (3.4) and arguing as in Subsection 3.7, we believe that, putting $m_{j}:=\min \left\{\left|\tau_{j}-\varphi\right| \cdot\left|1+\varphi \tau_{j}^{\sigma}\right|,\left|\tau_{j}-(1+\varphi)\right| \cdot\left|1+\varphi \tau_{j}^{\sigma}-\varphi\right|,\left|\tau_{j}-\varphi\left(1+\tau_{j}\right)\right| \cdot\left|1+\tau_{j}^{\sigma}(1+\varphi)\right|\right\}$, for $j=1, \ldots, s$, we have

$$
c_{\tau}(\varphi)=\min _{1 \leq j \leq s} \frac{2 m_{j}}{\left(\tau_{j}-\tau_{j}^{\sigma}\right)\left(1+\varphi^{2}\right)}
$$

Establishing (3.8) seems to be a difficult problem.

\section{Further results}

In this section, we take the point of view of Diophantine approximation to discuss several questions naturally related to the quantity $h(\alpha)$ defined in (1.1).

We begin with a link between the constant $c_{\tau}(\xi)$ and the continued fraction expansions of $\xi$ and $\tau$.

Lemma 4.1. Let $\tau$ be a quadratic real number. Let $b_{1}, \ldots, b_{s}$ be the shortest period in its continued fraction expansion. Then, an irrational real number $\xi=\left[a_{0} ; a_{1}, a_{2}, \ldots\right]$ not in $\mathcal{E}_{\tau}$ satisfies $c_{\tau}(\xi)>0$ if, and only if, the infinite word $a_{0} a_{1} \ldots$ does not contain arbitrarily large repetitions neither of $b_{1} \ldots b_{s}$, nor of $b_{s} \ldots b_{1}$.

Proof. Assume first that the infinite word $a_{0} a_{1} \ldots$ contains arbitrarily large repetitions of $b_{1} \ldots b_{s}$ and let $K_{\tau}$ denote the denominator of the rational number $\left[0 ; b_{1}, \ldots, b_{s}\right]$. Let $m \geq 2$ and $r \geq 1$ be integers such that $a_{r+j s+i}=b_{i}$ for $i=1, \ldots, s$ and $j=0, \ldots, m-1$. Set $\alpha_{r}:=\left[a_{0} ; a_{1}, a_{2}, \ldots, a_{r}, \overline{b_{1}, \ldots, b_{s}}\right]$ and denote by $\left(p_{\ell} / q_{\ell}\right)_{\ell \geq 1}$ the sequence of its convergents. 
Since $\alpha_{r}$ and $\xi$ have (at least) their $r+m s$ first partial quotients in common, it follows from Lemma 2.4 that

$$
\left|\xi-\alpha_{r}\right|<q_{r+m s}^{-2} \leq q_{r}^{-2} K_{\tau}^{-2 m s},
$$

while Lemma 2.1 asserts that

$$
h\left(\alpha_{r}\right)<4 q_{r}^{2}
$$

Consequently, we deduce that

$$
\left|\xi-\alpha_{r}\right| \cdot h\left(\alpha_{r}\right)<4 K_{\tau}^{-2 m s} .
$$

Since $m$ can be taken arbitrarily large, we conclude that $c_{\tau}(\xi)=0$.

The converse is slightly more difficult to establish. Assume that $c_{\tau}(\xi)=0$ and let $\varepsilon$ be a positive real number. Set $B:=\max \left\{b_{1}, \ldots, b_{s}, 2\right\}$. There exists $\alpha$ in $\mathcal{E}_{\tau}$ such that

$$
|\xi-\alpha| \cdot h(\alpha)<\varepsilon
$$

It follows from Corollary 2.3 that, if $\varepsilon$ is sufficiently small, then there exist a cyclic permutation $c_{1}, \ldots c_{s}$ of $b_{1}, \ldots, b_{s}$ or of $b_{s}, \ldots, b_{1}$ and an integer $r$ such that

$$
\alpha=\left[a_{0} ; a_{1}, a_{2}, \ldots, a_{r}, \overline{c_{1}, \ldots, c_{s}}\right]
$$

with $a_{r} \neq c_{s}$. Denote by $\left(p_{\ell} / q_{\ell}\right)_{\ell \geq 1}$ the sequence of convergents of $\alpha$ and observe that, by Lemma 2.1,

$$
h(\alpha) \geq q_{r}^{2} /\left(4 B^{3}\right) .
$$

Let $m(\alpha)$ be the smallest integer for which the $m(\alpha)$-th partial quotients of $\xi$ and of $\alpha$ are different. Then, by Lemma 2.2, we get the lower bound

$$
|\xi-\alpha| \geq \frac{1}{12(B+2) q_{m(\alpha)}^{2}} .
$$

Since $q_{r+h} \leq(B+1)^{h} q_{r}$ for $h \geq 1$, it follows from (4.1), (4.2) and (4.3) that

$$
q_{r}^{2}<48 \varepsilon(B+2) B^{3} q_{m(\alpha)}^{2} \leq 48 \varepsilon(B+2) B^{3}(B+1)^{m(\alpha)-r} q_{r}^{2} .
$$

Since $\varepsilon$ can be taken arbitrarily small, this shows that $m(\alpha)-r$ can be arbitrarily large, thus, the infinite word $a_{0} a_{1} a_{2} \ldots$ contains arbitrarily large repetitions of the block $b_{1} \ldots b_{s}$ or of the block $b_{s} \ldots b_{1}$. This concludes the proof of the lemma.

In 1966, Schmidt $[13,14]$ developed his theory of $\alpha-\beta$ games and winning sets. The next result is an easy consequence of Lemma 4.1.

Theorem 4.2. For every quadratic real number $\tau$ the set

$$
\left\{\xi \in \mathbf{R}: c_{\tau}(\xi)>0\right\}
$$

has Lebesgue measure zero. It is a winning set, thus it has Hausdorff dimension 1. 
The first assertion of Theorem 4.2 follows from the well-known fact that every finite block of positive integers occurs in the continued fraction expansion of almost all real numbers.

Proof. Let $b_{1}, \ldots, b_{s}$ denote the shortest period of the continued fraction expansion of $\tau$. By Lemma 4.1, an irrational number $\xi$ satisfies $c_{\tau}(\xi)>0$ if, and only if, its continued fraction expansion, viewed as an infinite word, does not contain arbitrarily large repetitions of $b_{1} \ldots b_{s}$ or of $b_{s} \ldots b_{1}$. To win the game, the second player has simply to avoid certain intervals, as in Theorem 4 of [13], where it is proved that, under some assumption on $\alpha, \beta$ and on the integer $b \geq 2$, the set of real numbers having only finitely many digits 0 in their $b$-ary expansion is $(\alpha, \beta)$-winning. We omit the details.

For an irrational number $\xi$ and an integer $b \geq 2$, let $v_{b}(\xi)$ denote the supremum of the real numbers $v$ such that

$$
\left\|b^{n} \xi\right\|<\left(b^{n}\right)^{-v}
$$

for infinitely many positive integers $n$. Here, $\|\cdot\|$ denotes the distance to the nearest integer. The exponents of approximation $v_{b}$ have been recently introduced and studied in [4]. They detect large repetitions of digits 0 or of digits $b-1$.

Actually, given an integer $b \geq 2$ and a rational number $p / q$ with $0 \leq p / q<1$, we can as well define $v_{b, p / q}(\xi)$ to be the supremum of the real numbers $v$ such that

$$
\left\|b^{n} \xi+p / q\right\|<\left(b^{n}\right)^{-v}
$$

for infinitely many positive integers $n$. This means that we approximate $\xi$ by rational numbers whose $b$-ary expansion is ultimately periodic, with the same period as the $b$-ary expansion of $p / q$. Almost all real numbers $\xi$ satisfy $v_{b, p / q}(\xi)=0$.

A natural analogue (for restricted quadratic approximation) of the exponents $v_{b, p / q}$ (for restricted rational approximation) in the present setting is given by the exponents $v_{\tau}$ defined as follows.

Definition 4.3. For a quadratic number $\tau$ and an irrational number $\xi$ not in $\mathcal{E}_{\tau}$, we denote by $v_{\tau}$ the supremum of the real numbers $v$ such that

$$
|\xi-\alpha|<h(\alpha)^{-v}
$$

for infinitely many $\alpha$ in $\mathcal{E}_{\tau}$.

Note that the choice of an integer base $b$ corresponds to that of a continued fraction algorithm and the choice of a rational number $p / q$ corresponds to that of a quadratic number $\tau$. In the present text, we consider only the usual continued fraction algorithm.

It follows from Lemma 2.1 that every real number $\xi$ not in $\mathcal{E}_{\tau}$ satisfies $v_{\tau}(\xi) \geq 1$. Furthermore, almost all real numbers $\xi$ satisfy $v_{\tau}(\xi)=1$ and, for every real number $v \geq 1$, the Hausdorff dimension of the set

$$
\left\{\xi \in \mathbf{R}: v_{\tau}(\xi)=v\right\}
$$

is equal to $1 / v$; see also Theorem 1.3 of [9].

Our last result is a transcendence statement. 
Theorem 4.4. Let $\tau$ be a quadratic real number and $\xi$ an irrational number not in $\mathcal{E}_{\tau}$. If $v_{\tau}(\xi)>1$, then $\xi$ is transcendental.

Proof. Let $b_{1}, \ldots, b_{s}$ be the shortest period of the continued fraction expansion of $\tau$ and write $\xi=\left[a_{0} ; a_{1}, a_{2} \ldots\right]$. Let $\left(p_{\ell} / q_{\ell}\right)_{\ell \geq 1}$ be the sequence of partial quotients to $\xi$. Replacing $\tau$ by $-1 / \tau^{\sigma}$ if necessary, there exist, by assumption, a positive real number $\varepsilon$ and an infinite set $\mathcal{R}$ of positive integers such that, for any $r$ in $\mathcal{R}$, the quadratic number $\alpha_{r}=$ $\left[a_{0} ; a_{1}, \ldots, a_{r}, \overline{b_{1}, \ldots, b_{s}}\right]$ in $\mathcal{E}_{\tau}$ satisfies $a_{r+1} \neq b_{1}$ and

$$
\left|\xi-\alpha_{r}\right|<h\left(\alpha_{r}\right)^{-1-\varepsilon}
$$

Note that it follows from Lemma 2.1 that

$$
h\left(\alpha_{r}\right) \gg q_{r}^{2}
$$

where, as below, the numerical constant implied by $\gg$ only depends on $b_{1}, \ldots, b_{s}$. Furthermore, denoting by $m\left(\alpha_{r}\right)$ the smallest integer for which the $m\left(\alpha_{r}\right)$-th partial quotients of $\xi$ and of $\alpha_{r}$ are different, Lemma 2.2 implies that

$$
\left|\xi-\alpha_{r}\right| \gg q_{m\left(\alpha_{r}\right)}^{-2}
$$

The combination of (4.4), (4.5) and (4.6) gives then

$$
q_{m\left(\alpha_{r}\right)} \gg q_{r}^{1+\varepsilon}
$$

and we deduce from Lemma 2.4 that there exists a positive real number $\varepsilon^{\prime}$, depending only on $b_{1}, \ldots, b_{s}$, such that

$$
m\left(\alpha_{r}\right) \geq r+s\left\lfloor\varepsilon^{\prime} \log q_{r}\right\rfloor,
$$

for any sufficiently large integer $r$ in $\mathcal{R}$.

Then, arguing as in the proof of Theorem 3.2 of [2] (see also Theorem 1.3 of [7]), it follows from the Schmidt Subspace Theorem that $\xi$ is either transcendental or quadratic (we omit the details). But if $\xi$ is quadratic, it must be in $\mathcal{E}_{\tau}$. This proves the theorem.

Unlike in Theorem 3.2 of [2] (see also Theorem 1.3 of [7]), we do not need in Theorem 4.4 an assumption on the growth of the denominators of the convergents to $\xi$. Indeed, here and unlike in those papers, the number of times that the block $b_{1}, \ldots, b_{s}$ is repeated is not only at least equal to some absolute constant times $r$, but also at least equal to some absolute constant times $\log q_{r}$.

Acknowledgements: I am very pleased to thank Jouni Parkkonen and Frédéric Paulin for having encouraged me to write this note and for fruitful correspondence. Many thanks to the referee for a very careful reading. 


\section{References}

[1] B. Adamczewski and Y. Bugeaud, On the Littlewood conjecture in simultaneous Diophantine approximation. J. London Math. Soc. 73 (2006), 355-366.

[2] B. Adamczewski and Y. Bugeaud, On the Maillet-Baker continued fractions, J. reine angew. Math. 606 (2007), 105-121.

[3] B. Adamczewski and Y. Bugeaud, Transcendence measures for continued fractions involving repetitive or symmetric patterns, J. Europ. Math. Soc. 12 (2010), 883-914.

[4] M. Amou and Y. Bugeaud, Expansions in integer bases and exponents of Diophantine approximation, J. London Math. Soc. 81 (2010), 297-316.

[5] Y. Bugeaud, Approximation by algebraic numbers. Cambridge Tracts in Mathematics 160, Cambridge, 2004.

[6] Y. Bugeaud, Continued fractions with low complexity: Transcendence measures and quadratic approximation, Compos. Math. 148 (2012), 718-750.

[7] Y. Bugeaud, Automatic continued fractions are transcendental or quadratic, Ann. Sci. École Norm. Sup. To appear.

[8] G. H. Hardy and E. M. Wright. An introduction to the theory of numbers, 5th. edition, Clarendon Press, 1979.

[9] J. Parkkonen and F. Paulin, Spiraling spectra of geodesic lines in negatively curved manifolds, Math. Z. 268 (2011), 101-142.

[10] J. Parkkonen et F. Paulin, Équidistribution, comptage et approximation par des irrationnels quadratiques, J. Modern Dynamics 6 (2012), 1-40.

[11] J. Parkkonen and F. Paulin, Erratum to Spiraling spectra of geodesic lines in negatively curved manifolds. Preprint.

[12] O. Perron, Die Lehre von den Ketterbrüchen. Teubner, Leipzig, 1929.

[13] W. M. Schmidt, On badly approximable numbers and certain games, Trans. Amer. Math. Soc. 123 (1966), 178-199.

[14] W. M. Schmidt, Diophantine Approximation, Lecture Notes in Math. 785, Springer, Berlin, 1980.

Yann Bugeaud

Université de Strasbourg

Mathématiques

7, rue René Descartes

67084 STRASBOURG (FRANCE)

bugeaud@math.unistra.fr 